\section{Protective head-cooling during cardiac arrest and cardiopulmonary resuscitation: the original animal studies}

\author{
Eric W. Brader,' Dietrich Jehle, ${ }^{2}$ \\ Michael Mineo, ${ }^{2}$ Peter Safar ${ }^{3 *}$ \\ 'Department of Emergency Medicine, \\ Allegheny General Hospital, Pittsburgh, \\ PA; \\ 2State University of New York at Buffalo, \\ Department of Emergency Medicine, \\ Buffalo, NY; \\ ${ }^{3}$ University of Pittsburgh, Safar Center \\ for Resuscitation Research, Pittsburgh, \\ Pennsylvania, USA *deceased
}

\begin{abstract}
Prolonged standard cardiopulmonary resuscitation (CPR) does not reliably sustain brain viability during cardiac arrest. Pre-hospital adjuncts to standard CPR are needed in order to improve outcomes. A preliminary dog study demonstrated that surface cooling of the head during arrest and CPR can achieve protective levels of brain hypothermia $\left(30^{\circ} \mathrm{C}\right)$ within 10 minutes. We hypothesized that protective head-cooling during cardiac arrest and CPR improves neurological outcomes. Twelve dogs under light ketamine-halothane-nitrous oxide anesthesia were arrested by transthoracic fibrillation. The treated group consisted of six dogs whose shaven heads were moistened with saline and packed in ice immediately after confirmation of ventricular fibrillation. Six control dogs remained at room temperature. All 12 dogs were subjected to four minutes of ventricular fibrillation and 20 minutes of standard CPR. Spontaneous circulation was restored with drugs and countershocks. Intensive care was provided for five hours postarrest and the animals were observed for 24 hours. In both groups, five of the six dogs had spontaneous circulation restored. After three hours, mean neurological deficit was significantly lower in the treated group $(\mathrm{P}=0.016$, with head-cooled dogs averaging $37 \%$ and the normothermic dogs 62\%). Two of the six headcooled dogs survived 24 hours with neurological deficits of $9 \%$ and $0 \%$, respectively. None of the control group dogs survived 24 hours. We concluded that head-cooling attenuates brain injury during cardiac arrest with prolonged CPR. We review the literature related to the use of hypothermia following cardiac arrest and discuss some promising approaches for the pre-hospital setting.
\end{abstract}

\section{Introduction}

More than 340,000 Americans die each year of coronary heart disease in the pre-hospital setting. ${ }^{1}$ In addition, there are a large number of patients who sustain permanent neurological damage following successful resuscitation from cardiac arrest. Adjuncts to cardiopulmonary resuscitation (CPR) are needed to improve the neurological outcome of these patients. Application of protective therapy prior to restoration of circulation, such as hypothermia, may prevent the post-ischemic encephalopathy that follows cardiac arrest. One of the most important mechanisms by which therapeutic hypothermia prevents further damage to the brain is by decreasing the oxygen demand of cerebral tissue.

Hypothermia, whether intentional or by accident, has long been known to prevent brain damage during cardiac arrest. Hypothermia has also been employed after return of spontan-eous circulation with some encouraging results..$^{3,4}$ Instead of allowing hypothermia to be of value in only these rare patients, it was proposed that the application of hypothermia be examined as a protective tool during "routine" cardiac arrest. This study, completed in 1984, compared cardiac and cerebral resuscitability of normothermic versus head-cooled dogs subjected to a prolonged CPR insult. ${ }^{5}$ The authors hypothesized that protective head-cooling during cardiac arrest and CPR achieves protective levels of brain hypothermia and results in improved neurological outcomes.

This study was conducted initially in the early $1980 \mathrm{~s}$ and published as an abstract in $1985 .^{5}$ From the 1990s to the present there has been a regained interest in the topic with a flourishing of publications related to the topic. The result has been organizations such as the Canadian Resuscitation Council and the Canadian Association of Emergency Physicians releasing position statements advocating head-cooling for patients who present with non-perfusing ventricular fibrillation or ventricular tachycardia and are resuscitated to hemodynamic stability yet remain unresponsive. ${ }^{6,7}$ The optimal method of achieving cooling is still unclear, with multiple methods being applied. The data from this study, conducted over two decades ago, is being published here for both historical value and because the data specifically on head-cooling during cardiac arrest may contribute to this ongoing discussion.

\section{Materials and Methods}

A series of pilot experiments was performed to investigate cooling rates of the brain at room temperature and with head-cooling. The
Correspondence: Dietrich Jehle, State University of New York at Buffalo, Department of Emergency Medicine, 462 Grider Street, Buffalo, NY 14215, USA. E-mail: jehle@ecmc.edu

Key words: head-cooling, cerebral hypothermia, cerebral resuscitation, cardiopulmonary resuscitation.

Acknowledgements: the authors thank Dr. Fritz Sterz, from the University of Vienna, for his assistance in providing reference materials for the preparation of this manuscript. Support was generously granted by Allegheny-Singer Research Institute and the Safar Center for Resuscitation Research.

Received for publication: 9 December 2009.

Revision received: 29 January 2010.

Accepted for publication: 29 January 2010.

This work is licensed under a Creative Commons Attribution 3.0 License (by-nc 3.0).

(c) Copyright E.W. Brader et al., 2010

Licensee PAGEPress, Italy

Neurology International 2010; 2:e3

doi:10.4081/ni.2010.e3

formal experimental study was set up to investigate the role of head-cooling initiated at the onset of witnessed pre-hospital cardiac arrest. The experimental protocol was designed to replicate a witnessed cardiac arrest, with four minutes of ventricular fibrillation (VF), 20 minutes of basic life support (BLS), followed by advanced cardiac life support (ACLS). This study was approved by the institutional review board, and the Animal Care and Use Committee of the Allegheny-Singer Research Institute.

\section{Pilot study}

Several pilot experiments were preformed to investigate the feasibility of achieving protective levels of brain hypothermia within ten minutes of cardiac arrest, utilizing head surface cooling. Three dogs were prepared in a similar fashion to the formal study with the exception of having temperature probes, insulated and sealed with bone wax, placed in the cardiac portion of the esophagus, below the galea, in the rectum, and one centimeter into the right frontoparietal cerebral cortex. After transthoracic electrical induction of VF, Dog \#1 was allowed to cool undisturbed, with no CPR and no head-cooling, at room temperature, Dog \#2 was head-cooled after induction of $\mathrm{VF}$ but had no CPR performed, and Dog \#3 was head-cooled while CPR was administered for VF by a Michigan Instruments "thumper." A fourth undisturbed pilot study was performed with a prototype cooling device $\left(\mathrm{NH}_{4} \mathrm{NO}_{3}-\mathrm{H}_{2} \mathrm{O}\right.$; cold pack technology) and with probes in the thalamic, subcortical, and subgaleal regions. 


\section{Experimental study}

Twelve healthy flat-chested mongrel dogs weighing 12-25 $\mathrm{kg}$ were fasted overnight with water ad lib. All dogs were premedicated with ketamine $(10 \mathrm{mg} / \mathrm{kg} \mathrm{IM})$ and anesthetized with halothane $(0.75-4.0 \%)$, nitrous oxide (50\%), and oxygen (50\%). Endotracheal intubation was performed with a cuffed tube when anesthesia reached sufficient depth. Preinsult rectal and cardiac temperatures were maintained at $37-38^{\circ} \mathrm{C}$. Infra-diaphragmatic aortic catheters and Swan-Ganz catheters with temperature probes were placed by femoral cutdown. All dogs had their heads shaved. The level of anesthesia was allowed to lighten by allowing all dogs to spontaneously breathe room air for 4-6 min. VF was induced transthoracically with 100 volts alternating current for 3 sec. Upon confirmation of VF, six dogs were cooled by having bags of ice placed around their heads. All dogs were subjected to $4 \mathrm{~min}$ ventricular fibrillation and 20 min "controlled" CPR. Sixty chest compressions per minute and one interposed ventilation (TV= $25 \mathrm{~mL} / \mathrm{kg}$, F10 1.0) per five compressions were delivered using a Michigan Instruments "thumper." Initial systolic blood pressure during CPR was maximized but not allowed to exceed 70 $\mathrm{mmHg}$. Initial chest compression was limited to two inches after 1-2 min manual CPR to loosen the chest. Sandbags were used to stabilize the chest laterally. If systolic blood pressure was not maintained above $30 \mathrm{mmHg}$ after 10 min CPR, the animal was excluded. Restoration of total spontaneous circulation (ROSC) was achieved using the 1984 ACLS protocol (Figure 1) and the total number of ACLS steps was calculated. Mean arterial pressure was maintained above $60 \mathrm{mmHg}$ using a continuous epinephrine infusion and fluid administration titrated by pulmonary artery pressure and/or central venous pressure. Following ROSC, the ice bags were removed from the head and warming was achieved by heating pads applied to the body but not to the cold, wet head. One hour after ROSC, the weaning process was begun. All dogs received 4-5 hr intensive care. Then they were returned to their cages with supplemental oxygen and maintenance IVs. Neurological deficit scores (NDS) were obtained at 3,12 , and $24 \mathrm{hr}$ postresuscitation. A slightly modified version of the neurological scoring system of the Resuscitation Research Center was employed to assess neurological outcome (Figure 2). An NDS of $100 \%$ represented brain death. No neurological scoring was performed when cardiopulmonary decline prevented adequate neurological testing (Figure 3).

Data were analyzed using the Student's twotailed t-test for independent means, unless otherwise referenced.

\section{Results}

\section{Pilot study}

In the pilot studies, therapeutic levels of cortical hypothermia were reached within $10 \mathrm{~min}$ utes $\left(<32^{\circ} \mathrm{C}\right)$ in head-cooled dogs (ice) whether CPR was applied or not (Figure 4A). Extremely hypothermic subgaleal temperatures $\left(26^{\circ} \mathrm{C}\right)$ were achieved within 10 minutes in instrumented animals with an ice pack technology (ammonium nitrate) cooling device (Figure 4B).

\section{Experimental study}

Six preliminary trials of the experimental study were initially carried out to formalize the protocol. All of the following 12 animals that were fibrillated were able to achieve ROSC and met inclusion criteria.

Head-cooled dogs had significantly better neurological outcomes than control dogs $(\mathrm{P}=0.016)$. Mean NDS for head-cooled dogs at three hours was $37.2 \%$ while mean NDS for control dogs was $61.8 \%$. Two head-cooled dogs survived 24 hours. One was neurologically intact while the other had an NDS of $9 \%$. No control dogs survived 24 hours. One control dog had life support terminated after brain death, as defined by NDS (Figure 2), at 18 hours. With the exception of the previously mentioned dog, all dogs who failed to survive 24 hours died of cardiopulmonary causes.

In spite of statistically significantly lower core temperatures at the time of defibrillation $(\mathrm{P}=0.04$; one-tailed $\mathrm{t}$-test), head-cooled dogs had the same ease of cardiac resuscitation. In addition, mean ACLS steps required to restore spontaneous circulation was not significantly different between head-cooled and control dogs $(\mathrm{P}=0.713 ; 5$ vs. 5.7 ACLS steps, respectively). In both groups, five of the six dogs had successful ROSC. Mean arterial pressure obtained during CPR was not significantly different $(\mathrm{P}=0.262)$ between head-cooled (25.50 $\mathrm{mmHg})$ and control (19.85 mmHg) groups.

In head-cooled dogs, core temperature became significantly lower than rectal temperature $(\mathrm{P}<0.001)$ one minute after ROSC and remained so $(\mathrm{P}=0.004)$ at five minutes after ROSC (Figure 5). The nadir of afterdrop, that is drop in cardiac temperature after ROSC, occurred one minute after ROSC and averaged $1.6^{\circ} \mathrm{C}$ with core temperature falling to $34.5^{\circ} \mathrm{C}$. In control dogs, core and rectal temperatures never became significantly different $\left(<0.25^{\circ} \mathrm{C}\right.$ difference at 20 min of CPR). Rewarming was accomplished in one hour. There was no statistical difference in weight $(\mathrm{P}=0.442)$, core $(\mathrm{P}=0.619)$ and rectal $(\mathrm{P}=0.941)$ temperature, conjunctival oxygenation $(\mathrm{P}=0.945)$, and arterial pressure $(\mathrm{P}=0.968)$ between the groups at baseline.

\section{Discussion}

Cardiac arrest results in cerebral hypoperfusion with resultant global brain ischemia. In addition to the immediate damage caused by hypoxemia, there is a cascade of injuries

\begin{tabular}{|c|c|}
\hline 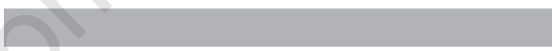 & ACLS Step \\
\hline \multicolumn{2}{|l|}{0 Minutes } \\
\hline 1. $\mathrm{HCO}_{3}-1 \mathrm{mEq} / \mathrm{Kg} \mathrm{IV}$ & $\# 1$ \\
\hline 2. Epi $1 \mathrm{mg} I V$ & $\# 1$ \\
\hline 3. $\mathrm{D}_{5} 1 / 2 \mathrm{NSS}-10 \% \mathrm{EBV}$ over 15 minutes IV & $\# 1$ \\
\hline 4. CPR for 1 minute - then CS with 200J if in VF & $\# 2$ \\
\hline 5. CS at 200J if still in VF after 1st CS & $\# 3$ \\
\hline \multicolumn{2}{|l|}{5 Minutes } \\
\hline 1. Epi $1 \mathrm{mg}$ IV & $\# 4$ \\
\hline 2. Draw ABG & $\# 4$ \\
\hline 3. CS at $200 \mathrm{~J} \times 2$ & $\# 5$ \#6 \\
\hline \multicolumn{2}{|l|}{10 Minutes } \\
\hline 1. $\mathrm{HCO}_{3}$ - by $\mathrm{ABG}$ if available or $0.5 \mathrm{mg} / \mathrm{kg}$ & $\# 7$ \\
\hline 2. Epi $1 \mathrm{mg}$ IV & $\# 7$ \\
\hline 3. Lidocaine $1 \mathrm{mg} / \mathrm{kg}$ IV bolus & $\# 7$ \\
\hline $4 \mathrm{CS}$ at $200 \mathrm{~J} \times 2$ & $\# 8$ \#9 \\
\hline \multicolumn{2}{|l|}{15 Minutes } \\
\hline 1. Epi $1 \mathrm{mg}$ IV & $\# 10$ \\
\hline 2. CS at $200 \mathrm{~J} \times 2$ & \#11 \#12 \\
\hline
\end{tabular}

*If no spontaneous circulation - terminate study; $* *$ If asystole or EMD - give Epi, HCO3 - CPR as above and atropine $0.5 \mathrm{mg}$ IV for asystole or $\mathrm{EMD}<60 / \mathrm{min} ;$ give no CS or lidocaine.

Figure 1. Advanced cardiac life support protocol in use in 1984. 
1. Level of Consciousness

$$
\begin{aligned}
0 & =\text { Normal } \\
30 & =\text { Clouded } \\
45 & =\text { Delirium } \\
60 & =\text { Stupor } \\
100 & =\text { Coma }
\end{aligned}
$$

2. Respiration

$$
\begin{aligned}
0 & =\text { Normal } \\
25 & =\text { Hyperventilate } \\
50 & =\text { Severe hyperventilation } \\
100 & =\text { Apnea or near apnea }
\end{aligned}
$$

3. Cranial nerve function

$$
\text { Pupil size }
$$

$0=$ Normal (4-7mm diameter)

2 = Abnormal (8mm diameter)

$5=$ Severe R/L side abnormal

Light reflex

$0=$ Normal

$2=$ Sluggish or R/L side variable

$5=$ Absent

Eye Position

$0=$ Normal

2 = Moderately abnormal

5 = Severely Abnormal

4. Lid reflex

$$
\begin{aligned}
& 0=\text { Normal } \\
& 2=\text { Sluggish } \\
& 5=\text { Absent }
\end{aligned}
$$

5. Corneal Reflex

$$
\begin{aligned}
& 0=\text { Normal } \\
& 2=\text { Sluggish R/L side } \\
& 5=\text { Absent }
\end{aligned}
$$

6. Oculocephalic reflex

$$
\begin{aligned}
0 & =\text { Normal (quick return) } \\
5 & =\text { Abnormal (slow return) } \\
10 & =\text { Absent }
\end{aligned}
$$

7. Menace reflex

$$
\begin{aligned}
0 & =\text { Normal (present) } \\
5 & =\text { Sluggish } \\
10 & =\text { Absent }
\end{aligned}
$$

8. Auditory response

$$
\begin{aligned}
0 & =\text { Normal } \\
5 & =\text { Sluggish } \\
10 & =\text { No Response }
\end{aligned}
$$

9. Gag Reflex

$$
\begin{aligned}
0 & =\text { Normal } \\
5 & =\text { Sluggish } \\
10 & =\text { Absent }
\end{aligned}
$$

10. Carinal Reflex

$$
\begin{aligned}
0 & =\text { Normal } \\
5 & =\text { Sluggish } \\
10 & =\text { Absent }
\end{aligned}
$$

11. Position

$$
\begin{aligned}
0 & =\text { Normal } \\
10 & =\text { Mildly Abnormal } \\
25 & =\text { Severely Abnormal }
\end{aligned}
$$

12. Muscle Tone

$$
\begin{aligned}
0 & =\text { Normal } \\
10 & =\text { Mildly Abnormal } \\
25 & =\text { Very Abnormal }
\end{aligned}
$$

13. Paralysis

$$
\begin{aligned}
0 & =\text { Normal Response/Reflexes } \\
10 & =\text { Some Movement } \\
25 & =\text { Abnormal }
\end{aligned}
$$

14. Pain Stimuli

$$
\begin{aligned}
0 & =\text { Normal Response } \\
10 & =\text { Sluggish } \\
25 & =\text { No Response }
\end{aligned}
$$

15. Feeding/Drinking

$$
\begin{aligned}
0 & =\text { Normal } \\
15 & =\text { Abnormal }
\end{aligned}
$$

16. Chewing

$$
\begin{aligned}
0 & =\text { Normal } \\
15 & =\text { Cannot Chew }
\end{aligned}
$$

17. Sitting

$$
\begin{aligned}
0 & =\text { Normal } \\
15 & =\text { Cannot Sit }
\end{aligned}
$$

18. Standing

$$
0=\text { Can Stand }
$$$$
15 \text { = Cannot Stand }
$$

19. Walking

$$
0=\text { Can walk without ataxia }
$$

15 = Walk with ataxia

$30=$ Cannot walk

20. Cleaning

$0=$ Can clean self

$10=$ Cannot clean self

Maximum score 500 points $=100 \%$ neurological deficit $=$ brain death. When testing motor function and behavior, disregard functional impairment caused by cutdowns.

Figure 2. Canine neurological deficit scoring.

induced by reperfusion. Reperfusion injuries are mediated by several mechanisms including protein synthesis inhibition, induction of proteases, and excitatory amino acid release. ${ }^{8}$

This study consisted of a dog model that simulated pre-hospital cardiac arrest with four minutes of down time prior to initiation of 20 minutes of standard CPR (BLS). Head-cooling was started at the onset of arrest and was continued during CPR, followed by ACLS. Headcooled dogs had significantly better neurologi- cal outcomes than control dogs three hours after spontaneous return of circulation. These results have been bolstered further by several subsequent studies that reinforce the premise that there are benefits of induced hypothermia in cardiac arrest. ${ }^{2,-12}$ Leonov, et al. also reported that mild hypothermia induced after the onset of cardiac arrest improves neurological performance, function, and overall brain morphology. ${ }^{9}$

While several mechanisms may play a role, head-cooled dogs demonstrated awareness at three hours post-arrest while no control dogs did. Although longer, more controlled postresuscitation studies are needed, it is felt that the superior three-hour NDS of head-cooled dogs is a valid indication of cerebral protection and insult attenuation. It has also been postulated that profound subgaleal cooling may redirect blood flow from the external to the internal carotid system, thus improving the efficacy of CPR in generating blood flow to the brain.

Regional rates of cooling have been examined in a cold water drowning model in which canines were submerged in cold water with clamped endotracheal tubes. Rate of cortical cooling after arrest in this experiment was found to be comparable to the rate found in our feasibility study. Cortical temperatures were found to drop faster than cardiac temperatures, as would be expected if conduction were the dominant mechanism of cooling. No diving reflex was noted to occur. ${ }^{13}$ These findings suggest that conductive cooling may be sufficient to account for cerebral protection in cold water drowning. As in accidental and intentional hypothermia, mild afterdrop was found to occur in head-cooled dog resuscitations. Apparently afterdrop was not of sufficient magnitude to trigger rescue arrest as none was noted to occur.

A limitation of this study is the use of sodium bicarbonate as part of the ACLS protocol. These experiments were conducted originally in 1984, and at that time sodium bicarbonate was utilized in the ACLS protocol. However, there is some recent data suggesting sodium bicarbonate is beneficial in prolonged cardiac arrest. ${ }^{14}$ Researchers also could not be blinded as to which dogs were in the head-cooled group or the control group. Objective NDS scoring scales were used in an attempt to minimize scoring biases. In addition, the study could have benefited from longer observation periods and histological examination of the brain at autopsy.

\section{Review of the literature}

The therapeutic use of hypothermia was described as early as the times of the Egyptians, Greeks, and Romans..$^{15}$ Napoleon's surgeon-general reported that wounded soldiers placed closer to the fire died quicker than soldiers not warmed. ${ }^{16}$ In the modern era, therapeutic hypothermia was described in a series of case reports in 1958 by a surgeon who cooled patients $\left(30-34^{\circ} \mathrm{C}\right)$ with signs of severe neurological injury after cardiac arrest. Three of these patients recovered completely while one had a moderate neurological deficit after recovery. ${ }^{17}$ Other case reports have described both intentional and unintentional hypothermia enabling patients to remain neurologically intact after one hour of total circulatory arrest in pediatric open heart surgery, ${ }^{18}$ after 
forty minutes of submersion in cold water, ${ }^{19}$ and after three-and-a-half hours of external CPR. ${ }^{10}$

The topic of therapeutic hypothermia in the treatment of cardiac arrest has regained interest in the late 1990s. In 1997, Bernard, et al. published a report in which they prospectively studied patients who suffered out-of-hospital cardiac arrest and remained unconscious after return of spontaneous circulation. They surface-cooled these patients in the ED and ICU for twelve hours and compared their neurological outcome with a control group of similar patients studied by retrospective chart review. They concluded that neurological outcome was significantly improved and mortality decreased in the mild hypothermic group with no increase in complications..$^{20} \mathrm{~A}$ similar 1998 study by Yanagawa, et al. surface-cooled patients for 48 hours and demonstrated a trend, although not statistically significant, toward increased survivability with mild hypothermia. Their cooled patients had an increased incidence of pulmonic complications, but they theorized this was because of extended (five to six days) use of paralytic and sedative agents. ${ }^{21}$ In 2000, the Hypothermia After Cardiac Arrest (HACA) Study Group published a prospective study on the feasibility and safety of therapeutic hypothermia after cardiac arrest. ${ }^{12}$ They cooled patients in the ED after out-of-hospital cardiac arrest using headcooling and external body cooling blankets, maintained these patients in mild hypothermia for 24 hours, followed by passive re-warming. They concluded that these patients had a two-fold improvement in outcome as compared to historical normothermic controls, without increasing the incidence of sepsis, coagulopathy, neutropenia, or thrombocytopenia. Yanagawa, et al. suggested that even patients with out-of-hospital cardiac arrest and out-of-hospital ROSC without witnessed arrest, bystander CPR, or initial rhythm of ventricular asystole may benefit from induced hypothermia. ${ }^{22}$

Although there is general agreement that mild hypothermia is most beneficial while deep hypothermia can be detrimental, ${ }^{23,24}$ different studies have used alternative methods that achieve hypothermia at different rates. Animal studies have shown that a fifteen minute delay in cooling can negate the beneficial effects of mild hypothermia. ${ }^{25}$ The HACA Study Group of 2000 took an average of 287 minutes to achieve adequate hypothermia, ${ }^{12}$ and the study by Feldberg, et al. required an average of 301 minutes to adequately cool patients, despite using both cooling blankets and iced gastric saline lavage. ${ }^{26}$ Feldberg and colleagues concluded that externally induced mild hypothermia is both slow and imprecise. Hachimi-Idrissi, et al. utilized a helmet device to achieve mild hypothermia post-ROSC in patients with cardiac arrest who remained
Neurological Deficit Scoring (NDS):

ND SCORING SUMMARY:

\begin{tabular}{lcccc} 
& \multicolumn{2}{c}{ Points } & \multicolumn{2}{c}{ Scores \% } \\
& Best & Worst & Best & Worst \\
1) Level of Consciousness & 0 & 100 & 0 & 20 \\
2) Respiration & 0 & 100 & 0 & 20 \\
3) Cranial Nerve Function & 0 & 100 & 0 & 0 \\
4) Motor and Sensory Function & 0 & 100 & 0 & 20 \\
5) Behavior & 0 & 100 & 0 & 20 \\
TOTAL & 0 & 500 & 0 & $100 \%$ \\
\hline
\end{tabular}

Figure 3. Neurological scoring system. Neurological deficit scoring carried out as per protocol, using modifications of the methods of Nemoto, et al. and Grisvold, et al. Clinical neurological results expressed as neurological deficit (ND) score in percent. ND $100 \%$ means brain death and $0 \%$ means normal.
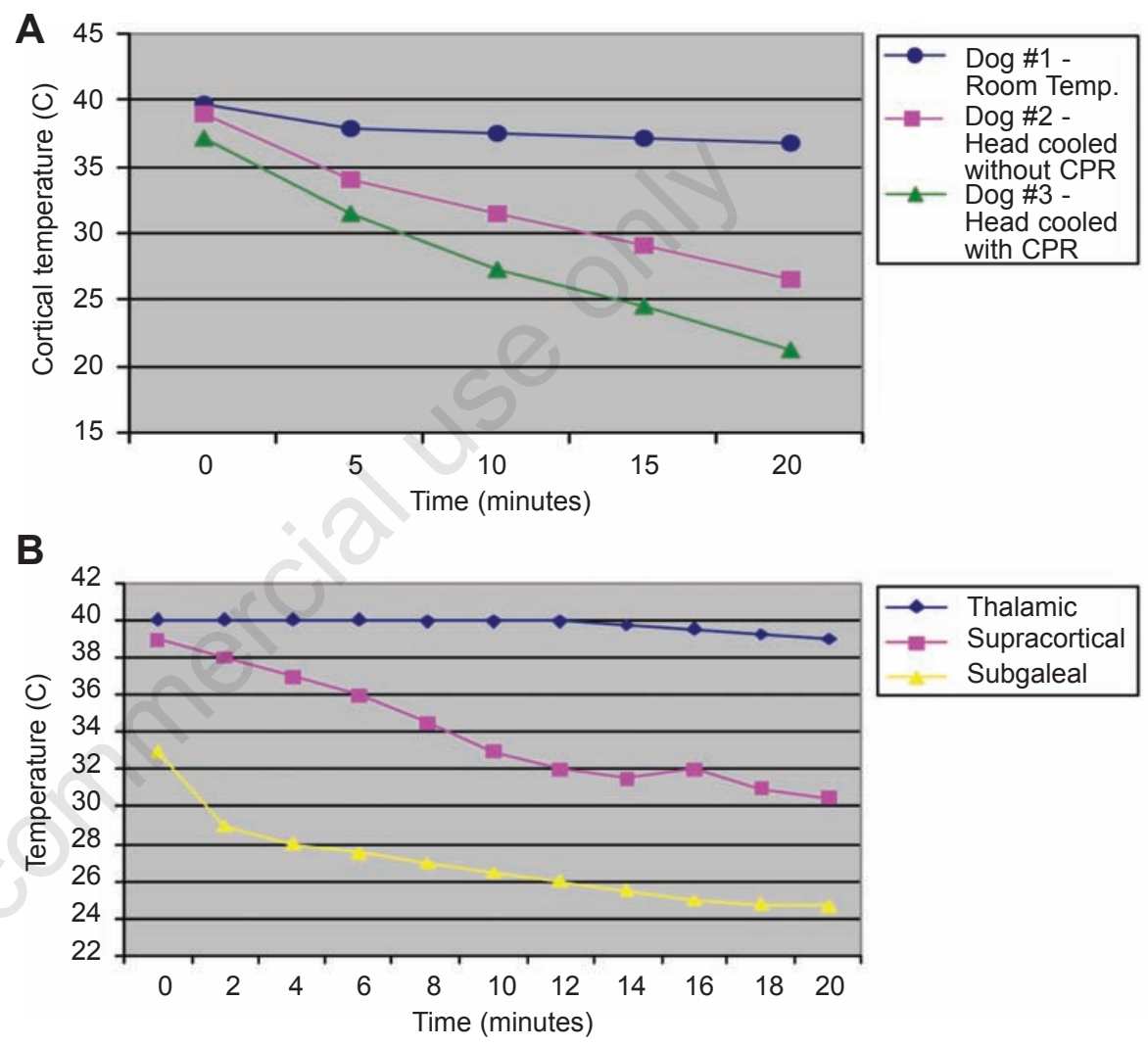

Figure 4. (A) Rates of cortical cooling. (B) Rates of cooling by regions of the brain without cardiopulmonary resuscitation.

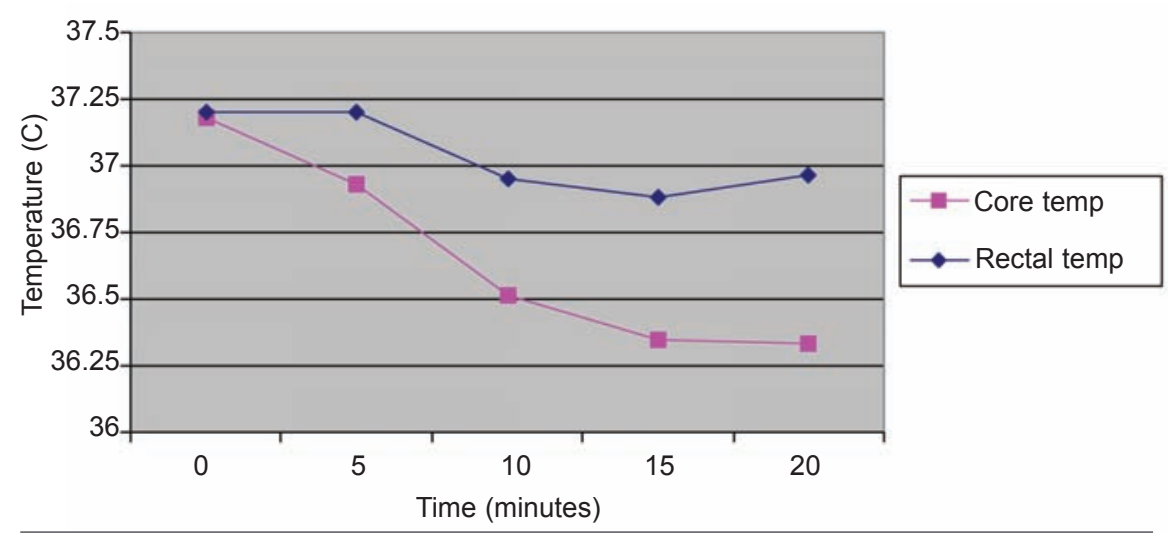

Figure 5. Core temperature (Swan-Ganz) vs. rectal temperature for head-cooled group. 


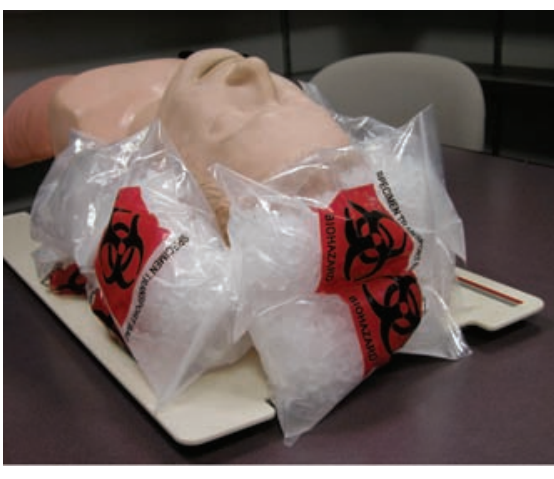

Figure 6. Ice packs to the head.

unconscious after return of spontaneous circulation. They were able to achieve a tympanic temperature of $34^{\circ} \mathrm{C}$ in a median of sixty minutes while it took a median of 180 minutes to achieve mild core hypothermia. ${ }^{27}$ Nozari, et al. attained tympanic temperatures of $34^{\circ} \mathrm{C}$ after approximately 20 minutes utilizing venovenous shunt cooling in an animal model. ${ }^{28} \mathrm{Kim}$, et $a l$. were able to rapidly reduce core temperature by $1.7^{\circ} \mathrm{C}$ in chemically paralyzed patients by rapid infusion of $2 \mathrm{~L}$ of $4^{\circ} \mathrm{C}$ normal saline..$^{29}$

The plethora of pilot and feasibility studies in the late 1990 s and early 2000 s led to two randomized controlled studies published in the New England Journal of Medicine in February 2002. The study by Bernard et al. compared moderate hypothermia to normothermia in patients who remained unconscious after resuscitation from out-of-hospital cardiac arrest. ${ }^{11}$ Patients were randomized to normothermia or hypothermia based on day of the week, and hypothermic patients were cooled in the field and later in the ED using cold packs applied to the head and torso. They were maintained moderately hypothermic for twelve hours, and then were actively re-warmed starting at eighteen hours. The researchers concluded that moderate hypothermia for twelve hours after resuscitation resulted in a significant improvement in outcome, but not a significant improvement in mortality. Therapeutic hypothermia was not associated with clinically significant adverse effects.

The study by the HACA Study Group analyzed the use of mild hypothermia in improving neurological recovery after resuscitation from out-of-hospital cardiac arrest. ${ }^{12}$ Patients who met the inclusion criteria and were brought to a participating ED were randomized via a sealed envelope to either the mild hypothermia or control group. Patients in the mild hypothermia group were cooled using an external cooling device and maintained mildly hypothermic for twenty-four hours followed by passive re-warming. The patients in the mild hypothermic group had both a significant decreased mortality rate and improved neurological outcome as compared with standard life support. There was not a significant difference in complication rate between the groups, although there was a non-statistically significant higher incidence of sepsis in the hypothermic group.

\section{Recommendations}

Based on these promising studies, the ALS Task Force of the International Liaison Committee on Resuscitation (ILCOR) recommends that unconscious adult patients with spontaneous circulation after out-of-hospital cardiac arrest should be cooled to $32-34^{\circ} \mathrm{C}$ for 12-24 hours when the initial rhythm is ventricular fibrillation. ${ }^{6,30}$ Although they make no specific recommendation in terms of methodology to achieve mild hypothermia, a variety of cooling methods have been applied in the past. These include cooling blankets, direct application of ice, iced saline gastric lavage, venous cooling, and cooling during bypass. None of these methods has met the ideal criteria of ease of use in the field along with quickly reaching mildly hypothermic temperatures. There are several very promising cooling techniques for use in the pre-hospital setting: including ice packs (Figure 6), cooling helmets, ${ }^{27}$ and cold IV fluid infusion. ${ }^{29,31}$

\section{Conclusions}

Cooling of the head is advantageous over systemic surface-cooling by limiting the drop in cardiac temperature prior to return of spontaneous circulation and its ease of application. Head-cooling can be applied clinically with a device employing cold pack technology that is portable, inexpensive, and applicable by laymen in the field. This study shows that headcooling is a promising means of protecting the brain during CPR that does not have an adverse effect on cardiac resuscitation.

\section{References}

1. American Heart Association. Heart Disease and Stroke Statistics, 2006 Update. Retrieved March 30, 2006 from http://circ.ahajournals.org/cgi/content/shor $\mathrm{t} / 113 / 6 / \mathrm{e} 85$

2. Safar P, Xiao F, Radovsky A, et al. Improved cerebral resuscitation from cardiac arrest in dogs with mild hypothermia plus blood flow promotion. Stroke 1996;27:105-13.

3. Wolfe KB. Effect of hypothermia on cerebral damage resulting from cardiac arrest. Am J Card 1960;6:809-12.

4. Rosomoff HL, Shulman K, Raynor R, et al. Experimental brain injury and delayed hypothermia. Surg Gynecol Obstet 1960;
110:27-32.

5. Brader E, Jehle D, Safar P. Protective head cooling during cardiac arrest in dogs. Ann Emerg Med 1985:14:510.

6. European Resuscitation Council. ERC Guidelines 2005. Retrieved March 30, 2006. http:/www.erc.edu/index.php/guidelines_download_2005/en/?

7. Canadian Association of Emergency Physicians. Position statement and guidelines for the use of hypothermia after cardiac arrest. http://www.caep.ca/002.policies/002-01.guidelines/guidelinesdocs/HypothermiaPositionStatement2005e.pdf

8. Angelos MG, Menegazzi JJ, Callaway, CC. Bench to bedside: resuscitation from prolonged ventricular fibrillation. Acad Emerg Med 2001;8:909-24.

9. Leonov Y, Sterz F, Safar P, et al. Mild cerebral hypothermia during and after cardiac arrest improves neurologic outcome in dogs. J Cerebral Blood Flow Metab 1990;10: 57-70.

10. Schissler P, Parker MA, Scott SJ Jr. Profound Hypothermia: value of prolonged cardiopulmonary resuscitation. South Med J 1981;74:474-7.

11. Bernard SA, Gray TW, Buist MJ, et al. Treatment of comatose survivors of out-ofhospital cardiac arrest with induced hypothermia. N Engl J Med 2002;346: 55763.

12. The Hypothermia after Cardiac Arrest Study Group. Mild therapeutic hypothermia to improve the neurologic outcome after cardiac arrest. N Engl J Med 2002; 346:549-56.

13. Tisherman S, Chabal C, Safar P, et al. Resuscitation of dogs from cold-water submersion using cardiopulmonary bypass. Ann Emerg Med 1985;14:389-96.

14. Vukmir RB, Katz L. Sodium Bicarbonate Study Group. Sodium bicarbonate Improves outcome in prolonged prehospital cardiac arrest. Am J Emerg Med 2006; 24:156-61.

15. Cool Heart Steering Committee. The CoolHeart ${ }^{\mathrm{TM}}$ Registry Mild Hypothermia in Cardiac Arrest. Retrieved April 22, 2004, from http://www.coolheart.com/materials/materials.html.

16. Larry IJ. Memoirs of Military Service and campaigns of the French Army. Vol 2. Baltimore: J Cushing, 1814, 156-64.

17. Williams GR, Spencer FC. The clinical use of hypothermia following cardiac arrest. Ann Surg 1958;148:462-8.

18. Dillard DH, Mohri H, Merendino KA. Correction of heart disease in infancy utilizing deep hypothermia and total circulatory arrest. J Thorac Cardiovasc Surg 1971; 61:64-9.

19. Siebke A, Rod T, Breivik H, et al. Survival 
after 40 minutes submersion without cerebral sequelae. Lancet 1975;1:1275-77.

20. Bernard SA, Jones BM, Horne MK. Clinical trial of induced hypothermia in comatose survivors of out-of-hospital cardiac arrest. Ann Emerg Med 1997;30:146-53.

21. Yanagawa $\mathrm{Y}$, Ishihara $\mathrm{S}$, Norio $\mathrm{H}$, et al. Preliminary clinical outcome study of mild resuscitative hypothermia after out-ofhospital cardiopulmonary arrest. Resusci 1998;39:61-6.

22. Yanagawa Y, Takasu A, Sakamoto T, et al. Indications and limitations of induced hypothermic therapy for out-of-hospital cardiopulmonary arrest. Am J Emerg Med 2006;24:214-6.

23. Weinrauch V, Safar P, Tisherman S, et al. Beneficial effects of mild hypothermia and detrimental effect of deep hypothermia after cardiac arrest in dogs. Stroke 1992; 23:1454-62.
24. Zeiner A, Holzer M, Sterz F, et al. Mild resuscitative hypothermia to improve neurological outcome after cardiac arrest: a clinical feasibility trial. Stroke 2000;31:86-94.

25. Kuboyama K, Safar P, Radovsky A, et al. Delay in cooling negates the beneficial effect of mild resuscitative cerebral hypothermia after cardiac arrest in dogs: a prospective randomized study. Crit Care Med 1993;21:1348-58.

26. Feldberg RA, Krieger DW, Chuang R, et al. Hypothermia after cardiac arrest: feasibility and safety of an external cooling protocol. Circulation 2001;104:1799-804.

27. Hachimi-Idrissi A, Corne L, Ebinger G, et al. Mild hypothermia induced by a helmet device: a clinical feasibility study. Resuscitation 2001;51:275-81.

28. Nozari A, Safar P, Stezoski SW, et al. Mild hypothermia during prolonged cardiopulmonary cerebral resuscitation increases conscious survival in dogs. Crit Care Med 2004;32:2110-6.

29. Kim F, Olsufka M, Carlbom D, et al. Pilot study of rapid infusion of $2 \mathrm{~L}$ of $4^{\circ} \mathrm{C}$ normal saline for induction of mild hypothermia in hospitalized, comatose survivors of outof-hospital cardiac arrest. Circulation 2005;112:715-9.

30. Nolan JP, Morley PT, Hoek TL, et al. Therapeutic hypothermia after cardiac arrest. An advisory statement by the Advancement Life Support Task Force of the International Liaison committee on Resuscitation. Resuscitation 2003;57:2315.

31. Bernard S, Buist M, Monteiro 0, et al. Induced hypothermia using large volume, ice-cold intravenous fluid in comatose survivors of out-of-hospital cardiac arrest: a preliminary report. Resuscitation 2003;56: 9-13. 